\title{
Effect of contact geometry on the contact stresses in a flat with rounded edge contact
}

\author{
Pankaj Dhaka, Raghu V. Prakash \\ Indian Institute of Technology Madras, India \\ pdbakanith@gmail.com, bttps://orid.org/0000-0001-7250-7845 \\ raghuprakash@iitm.ac.in, bttps://orcid.org/0000-0002-8888-022X
}

\begin{abstract}
The blade-disc dovetail interface in an aero-engine compressor is characterized by a non-uniform pressure distribution which can be obtained by an equivalent flat with rounded edge-on-plate configuration. The contact tractions for a mating pair are affected by many parameters which include, contact geometry, loading conditions, and material properties; with contact geometry being one of the prominent factors. In the present work, a 2-D elastic and elasto-plastic finite element analysis has been carried out for a rounded contact geometry to study the influence of the radius of the corners ' $R$ ' and length of the flat region ' $2 a$ '. It is observed that the peak tensile stress in the fretting direction decreases with increasing ' $a$ ' (for constant ' $R$ ') which is likely to delay the crack initiation. Also, as compared to elasto-plastic analysis, elastic analysis overestimates peak tensile stress and possibly gives a conservative estimate for the fretting fatigue life. Further, the effect of modelling elastic-plastic behaviour is significant for low $a / R$ ratio (for constant ' $R$ '). However, opposite trend was observed when ' $R$ ' was varied keeping ' $\mathrm{a}$ ' constant. Also, it is found that the effect of contact geometry cannot be characterized using a single parameter like $a / R$ ratio or contact area.
\end{abstract}

KEYwORDS. Dovetail; Flat with rounded edge; Fretting; Contact geometry.

\section{OPEN \\ (2) \\ ACCESS}

Citation: Dhaka, P., Prakash, R.V., Effect of contact geometry on the contact stresses in a flat with rounded edge contact, Frattura ed Integrità Strutturale, 48 (2019) 630-638.

Received: 04.01.2019

Accepted: 25.01.2019

Published: 01.04.2019

Copyright: (C) 2019 This is an open access article under the terms of the CC-BY 4.0 which permits unrestricted use, distribution, and reproduction in any medium, provided the original author and source are credited.

\section{INTRODUCTION}

I nvestigation of the contact behaviour is an indispensable exercise for many applications ranging from aero-engine and automotive components to orthopedic implants, hoists, power plant and electronic components [1-6]. The detrimental effect of a poor contact could be either loss of clearance in assemblies due to wear at the interface [2, 3 , 6] or complete failure due to crack initiation and propagation at the mating interface [1, 4]. The damage gets even more aggravated in the fretting regime where the sliding between the contact pair is of small amplitude (ranging between few microns to about 200 microns) because of smaller contact zone size which leads to highly localized stresses. The contact 
behaviour of a mating pair is affected by many parameters which include material properties, contact geometry, and loading conditions, with contact geometry being one of the crucial parameters. Contact geometry directly affects the completeness and conformality of a contact. In case of a complete contact, the nominal area of contact is fixed geometrically and is independent of normal load whereas, for an incomplete contact, the contact area increases with an increase in normal load. Further, if the largest characteristic dimension of the contact zone (contact radius) is considerably smaller than the smallest radius of the curvature of a mating pair, then the contact becomes non-conformal contact while vice-versa forms a conformal contact [7].

Most of the studies reported in the literature have generally focused on non-conformal and incomplete contacts viz. cylinder on plate configurations, with very few studies on conformal and complete contact. A flat with rounded edge-onplate contact is a type of conformal and partially complete contact. The relevance of analyzing flat with rounded edge contact lies in the fact that it is impractical to machine a flat surface with perfectly sharp corners owing to machining constraints which inevitably leads to corner radii. Another interesting application is in the case of an aero-engine compressor where the contact pressure distribution at the blade-disc dovetail interface can be represented by a flat-withrounded-edge geometry [8]. While in the case of a flat punch with sharp corners, singularity is present at contact edge and asymptotic contact pressure distribution is obtained, the rounding of the corners in a flat with rounded-edge leads to contact pressure falling to zero at the edge of the contact. This results in a non-uniform pressure distribution with constant pressure at the center and pressure peaks at the corners [9]. Ciavarella et al. [9-11] in one of their seminal works, proposed the analytical solution for the contact tractions in the case of partially flat contacts subjected to oscillating tangential and bulk load. Further, Warmuth et al. [12] observed that fretting in less conforming contacts leads to considerably higher wear rates compared to conformal contacts. They proposed that the difference in contact pressure because of different contact geometry was not the contributory factor for different wear rates rather it was attributed to the effect of contact geometry on debris flow in the contact zone. Many researchers $[13,14]$ studied the effect of radii of corners in the partially flat pad on fretting fatigue life and found that, in general, fretting fatigue life drops with an increase in corner radii which was attributed to increased stresses with rounding.

In the present work, effect of both half-length of central flat region ('a') and corner radii ('R') has been studied to understand its influence on the contact tractions and explore the possibility of quantifying the effect of contact geometry using a single parameter like a/R ratio or contact zone size. Finite element analysis was carried out for the both elastic and elasto-plastic case to understand the interrelation between yielding and contact geometry.

\section{METHODOLOGY}

$\mathrm{F}$ inite element methods tend to be quite advantageous in analyzing the contact problems which, otherwise are either infeasible to deal using experimental methods or prove to be cumbersome when subtle information regarding contact tractions is required. In the present work, a two-dimensional finite element analysis was carried out for a flat-with-rounded-edge pad on plate configuration. The geometric dimensions for flat-with-rounded-edge pad were taken from Fouvry et al. [9] which is a scaled-down model and represents the contact pressure distribution for a real blade-disc dovetail interface. The central flat region '2a' was taken as $3 \mathrm{~mm}$ while the radius at the corners(' $\mathrm{R}$ ') was $1.35 \mathrm{~mm}$. The plate was modelled with width 'l' and depth 'b' of $20 \mathrm{~mm}$ and $10 \mathrm{~mm}$ respectively. The model was meshed using 2-D plane strain, first order quadrilateral elements with reduced integration (CPE4R) and triangular elements (CPE3). These elements are generally preferred over second-order elements which give fluctuating pressure distribution for contact problems with friction [15]. The mesh was sufficiently refined in the contact zone and mesh size of $2 \mu \mathrm{m}$ was used while regions away from the contact zone were meshed using coarser mesh. The geometry and meshed model are shown in Fig. 1 (a) and 1 (b) respectively. The material behaviour for the flat with rounded edge pad and plate was modelled using Ti$6 \mathrm{Al}-4 \mathrm{~V}$ which is one of the widely used titanium alloys in aero-engines for fretting prone assemblies. The elasto-plastic behaviour of Ti-6Al-4V was modelled using Ramberg-Osgood law with material properties as given in Tab. 1 [16]. The Ramberg-Osgood deformation plasticity model available in ABAQUS ${ }^{\circledR}$ is generally used to describe elastic-plastic behaviour in applications pertaining to fracture mechanics and doesn't require separate definition for elastic and plastic segment of the stress-strain curve [17].

The Ramberg-Osgood coefficient and exponent were obtained by fitting a power law relationship in plastic strain vs. true stress response normalized with respect to corresponding yield point values using the following equation [18]:

$$
\frac{\varepsilon_{p}}{\varepsilon_{0}}=\alpha\left(\frac{\sigma}{\sigma_{0}}\right)^{n}
$$


where,

$\varepsilon_{p}$ is plastic strain,

$\sigma$ is true stress,

$\varepsilon_{0}, \sigma_{0}$ are yield point values for true stress and true strain respectively, and

$\alpha, n$ are Ramberg Osgood coefficient and exponent respectively

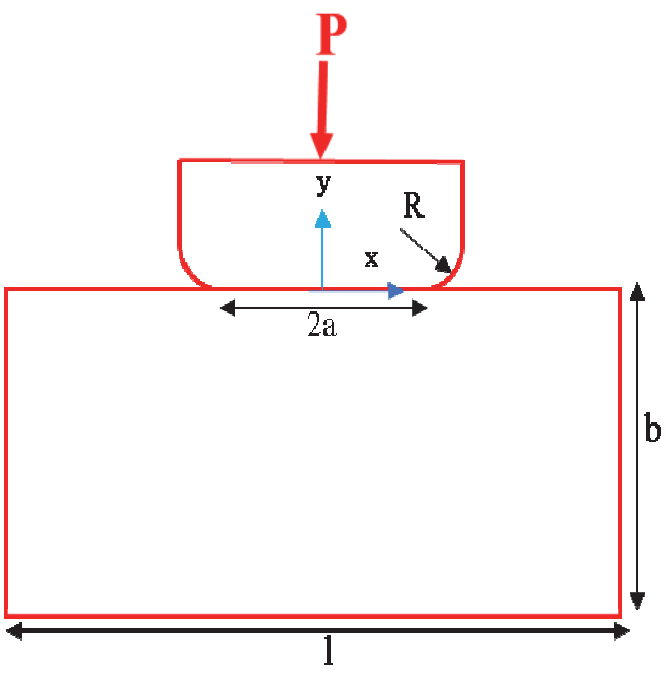

(a)

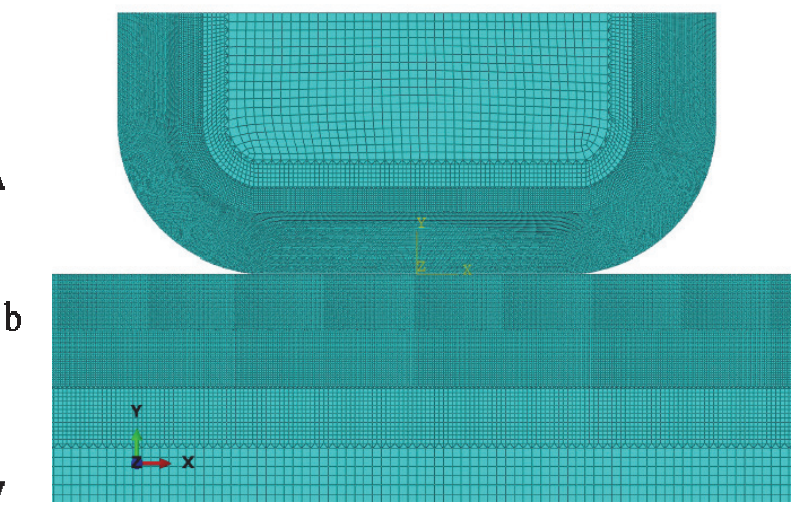

(b)

Figure 1: (a) Schematic of flat-with-rounded-edge on plate configuration, (b) Meshed model

\begin{tabular}{ccccc}
\hline Young's Modulus $(\mathrm{GPa})$ & Poisson's ratio & Yield strength $(\mathrm{MPa})$ & $\alpha$ & $\mathrm{n}$ \\
121 & 0.29 & 677.6 & 0.43 & 10 \\
\hline
\end{tabular}

Table 1: Material properties of Ti-6Al-4V.

\begin{tabular}{ccccccc}
\hline \multicolumn{5}{c}{ For validation of FEM } & \multicolumn{4}{c}{$\begin{array}{c}\text { Effect of contact geometry } \\
\text { Objective }\end{array}$} \\
S. No. & ' $a$ ' & 'R' & S. No. & 'R' \\
1 & 1.5 & 1.35 & 11 & Effect of 'a' & 1 & 1.35 \\
2 & 0.5 & 1.35 & 12 & & 2.7 & 1.35 \\
3 & 0.1 & 1.35 & 13 & & 4.05 & 1.35 \\
4 & 0.005 & 1.35 & 14 & Effect of 'R' & 1.5 & 1 \\
5 & 0.0005 & 1.35 & 15 & & 1.5 & 0.75 \\
6 & 1.5 & 0.135 & 16 & & 1.5 & 0.5 \\
7 & 1.5 & 2 & 17 & Effect of a/R & 1 & 2 \\
8 & 1.5 & 4 & 18 & ratio & 0.5 & 1 \\
9 & 1.5 & 6 & 19 & & 2 & 1 \\
10 & 1.5 & 8 & 20 & & 1 & 0.5 \\
\hline
\end{tabular}

Table 2: Analysis cases for the validation of finite element model

The frictional interaction between the fretting pad and the plate was defined using Coulomb's law of friction with a constant friction coefficient of 0.8; a value typically observed in experimental studies [8]. Combined Penalty (for shear traction) and augmented Lagrange (for normal contact) algorithm was used for contact formulation. The combined algorithm has been found particularly suitable for the fretting contact problems and computationally more efficient as compared to pure penalty formulation or pure Lagrange method [19]. A constant normal load (P) of $750 \mathrm{~N}$ has been 
applied on the top of the flat-with-rounded-edge pad and the pad is constrained to move in the vertical direction only. The bottom edge of the plate has been restrained from moving in both horizontal and vertical direction.

The validation of present finite element model was carried out using two approaches: first, the contact pressure distribution obtained from the elastic finite element analysis was compared with the analytical results proposed in the literature [10]; secondly, the length of the central flat region and corner radii were collapsed independently to make it tend towards the cylinder-on-plate and flat punch respectively. The results were compared with the analytical solution proposed by Hertz for cylinder-on-plate configuration [20,21]. Further, the effect of contact geometry was studied by varying either ' $a$ ', keeping ' $R$ ' as constant or by varying ' $R$ ', keeping 'a' as constant. The analysis was extended further to the elasto-plastic case to understand the interrelation between material yielding and contact geometry. All the analysis cases are tabulated in Tab. 2.

\section{RESULTS AND DISCUSSION}

he analysis results from the present study are presented in three different sections as follows:

\section{Finite Element Validation}

Fig. 2(a) shows the comparison between the contact pressure distribution obtained from finite element analysis and the analytical solution proposed by Ciavarella et al. [10]. It can be observed that the contact pressure distribution obtained from analytical and finite element analysis correlate well at the rounded corners but show significant deviation in the central contact zone. The possible reasons for the deviation could be: First, as the analytical solution proposed by Ciavarella et al. [10] is an approximate solution and involves calculation of many variables through an iterative approach which is likely to cause an error. Secondly, for higher values of a/R ratio, the half-plane idealization doesn't hold well. To further substantiate this argument and validate the present finite element model, the analysis was carried out with reduced values of ' $a$ '. Fig. 2(b) depicts the evolution of contact pressure distribution for different values of ' $a$ ', keeping ' $R$ ' as constant. It can be observed that, as the length of the central flat region is reduced keeping radii of corners as constant, the contact pressure distribution starts approaching towards that for a cylinder-on-plate configuration. Further, the solution agrees very well with the Hertz analytical solution [20] for cylinder-on-plate configuration.

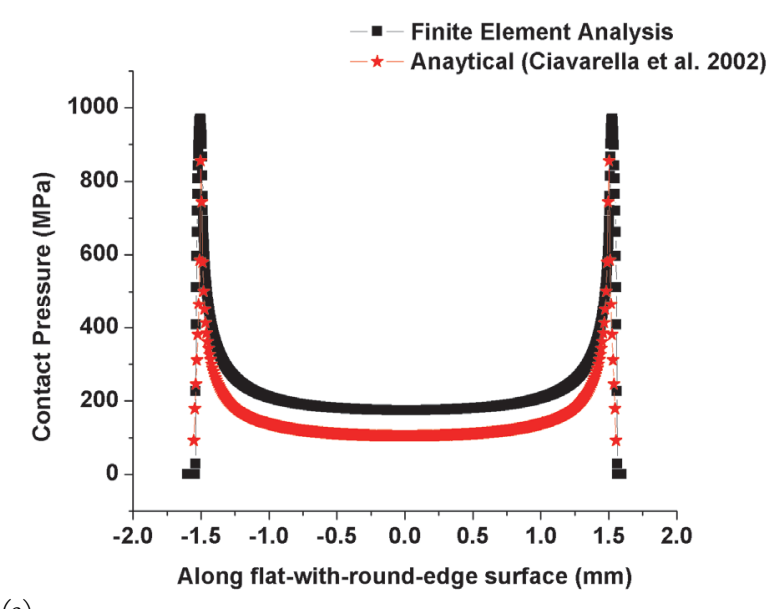

(a)

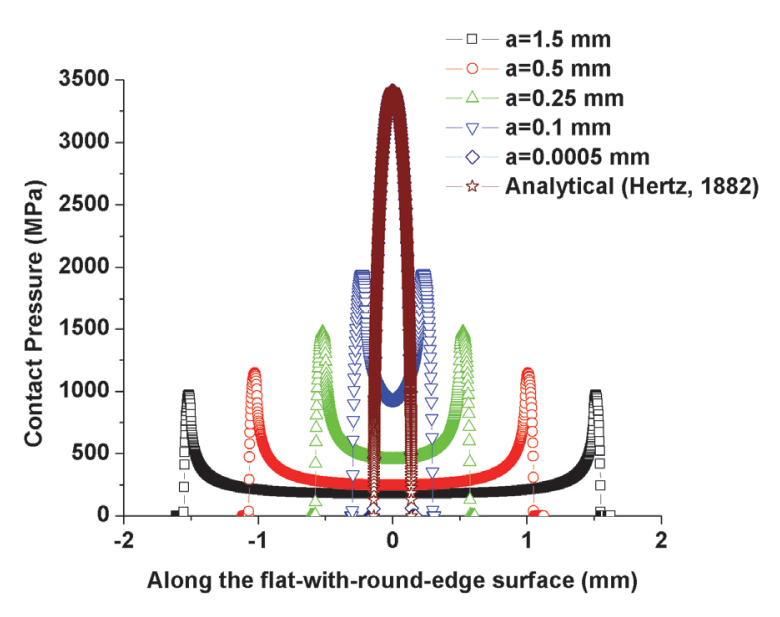

Figure 2: (a) Comparison between finite element analysis and analytical results, and (b) Evolution of contact pressure distribution with reducing 'a'.

Also, when the radius of corners was reduced, the contact pressure distribution tends towards that of the flat punch-onplate with the deviation from analytical results [21] being approximately $8 \%$ as shown in Fig. 3. 


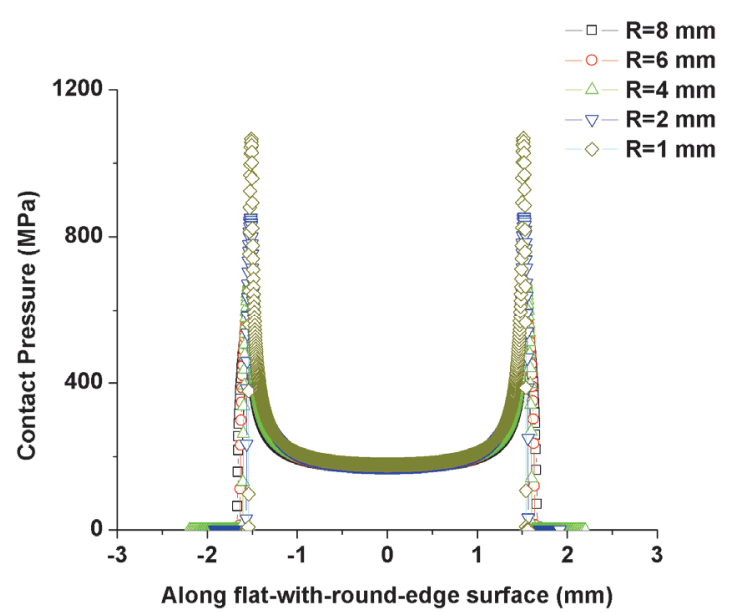

Figure 3: Evolution of contact pressure distribution with reducing ' $R$ '

\section{Effect of Contact Geometry}

The contact zone size in case of a flat-with-rounded-edge geometry is a function of two characteristic geometric dimensions viz. length of the flat region, ' $2 \mathrm{a}$ ' and corner radii, ' $\mathrm{R}$ ', in contrast to the case of a cylinder-on-plate configuration where radius of the cylinder is the only characteristic geometric dimension. To investigate whether the influence of contact geometry in case of a flat-with-rounded-edge geometry can be quantified using a single parameter like contact area or $\mathrm{a} / \mathrm{R}$ ratio, elastic finite element analyses were carried out for different contact geometries by varying ' $a$ ' and ' $\mathrm{R}$ ' independently.

From Fig. 4(a), it can be observed that the maximum contact pressure in the contact zone decreases with an increase in ' $a$ ' for a constant value of ' $R$ '. This is because of the proportional increase in the contact area with an increase in ' $a$ ' as shown in Fig. 4(b). Also, as 'a' tends towards zero, the maximum contact pressure approaches Hertzian pressure [20] for a cylinder with radius ' $R$ ', as marked with $\star$ in the Fig. 4(a).

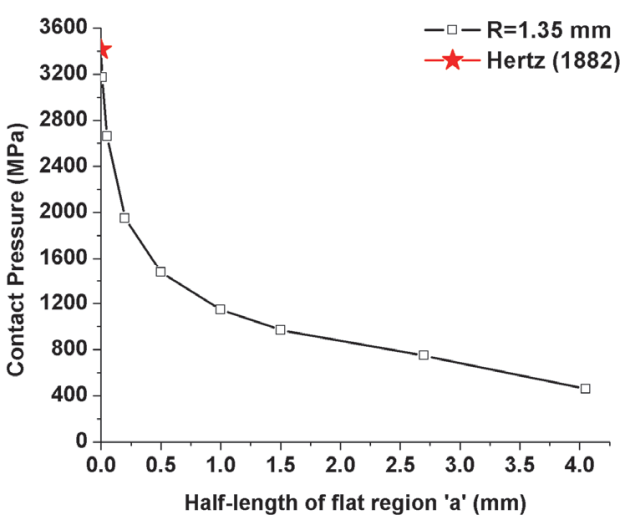

(a)

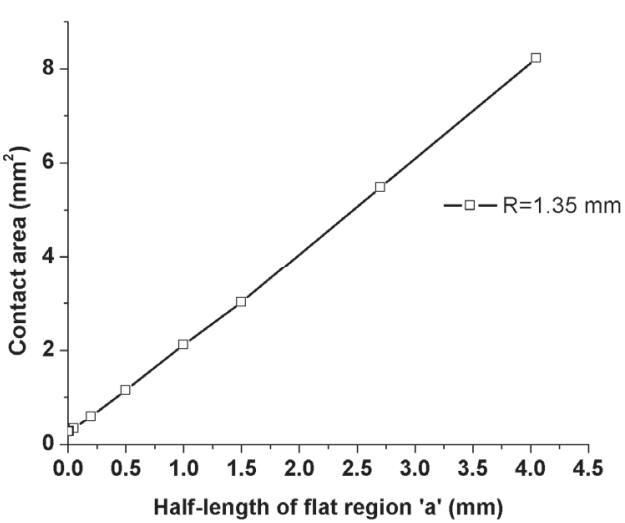

(b)

Figure 4: (a) Variation of maximum contact pressure with 'a', and (b) Contact zone size variation with 'a'

Further, the analysis was repeated by varying the value of 'R', keeping 'a' as constant. Fig. 5(a) shows the variation of maximum contact pressure, mean pressure in the central flat region and analytical mean pressure (i.e. $\mathrm{P} / 2 \mathrm{a}$ for unit thickness) with an increasing value of ' $R$ '. It can be observed that the maximum contact pressure decreases with an increase in ' $R$ ', tending towards a constant value for higher values of ' $R$ '. This can be explained by the evolution of contact zone size with ' $R$ ' which also shows a similar trend and starts saturating for the higher value of ' $R$ ' as shown in Fig. 5(b). Further, the mean contact pressure in the central flat region starts approaching theoretical value of mean pressure as corner radius is decreased below half-length of the flat region. Also, from the slope of the curves in Fig. 4(a), (b) and 5(a), (b), it can be inferred that effect of varying 'a' is more prominent on contact pressure and contact area than varying ' $R$ '.

Fig. 5 (c) shows the variation of maximum contact pressure with $a / R$ ratio for two different cases viz. for variable ' $a$ ', keeping ' $R$ ' as constant, and variable ' $R$ ', keeping ' $a$ ' as constant. It can be observed that both the curves intersect at a single point only i.e. approximately at $\mathrm{a} / \mathrm{R}=1$. Further, only single point of intersection was found between the curves of 
variable ' $a$ ' and variable ' $R$ ' when normalized contact pressure was plotted against the normalized contact area as shown in Fig. 5(d). The normalization of maximum contact pressure and contact area has been done with respect to maximum contact pressure and contact area obtained from the reference condition of $\mathrm{a}=1.5 \mathrm{~mm}$ and $\mathrm{R}=1.35 \mathrm{~mm}$. Thus, it can be inferred that, for a flat-with-rounded-edge geometry, the effect of contact geometry cannot be quantified using a single parameter like $\mathrm{a} / \mathrm{R}$ ratio or contact area, apart from the only case when both ' $\mathrm{a}$ ' and ' $\mathrm{R}$ ' are approximately equal.

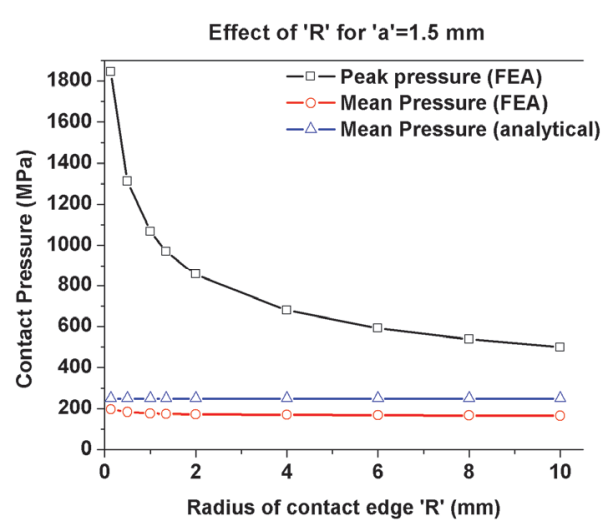

(a)

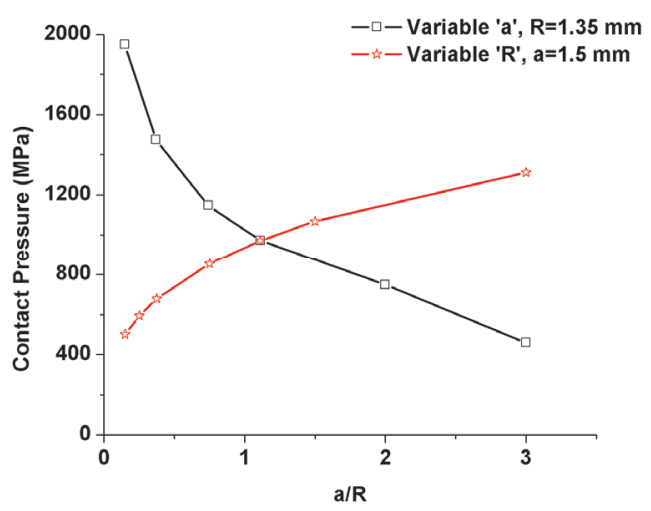

(c) (b)
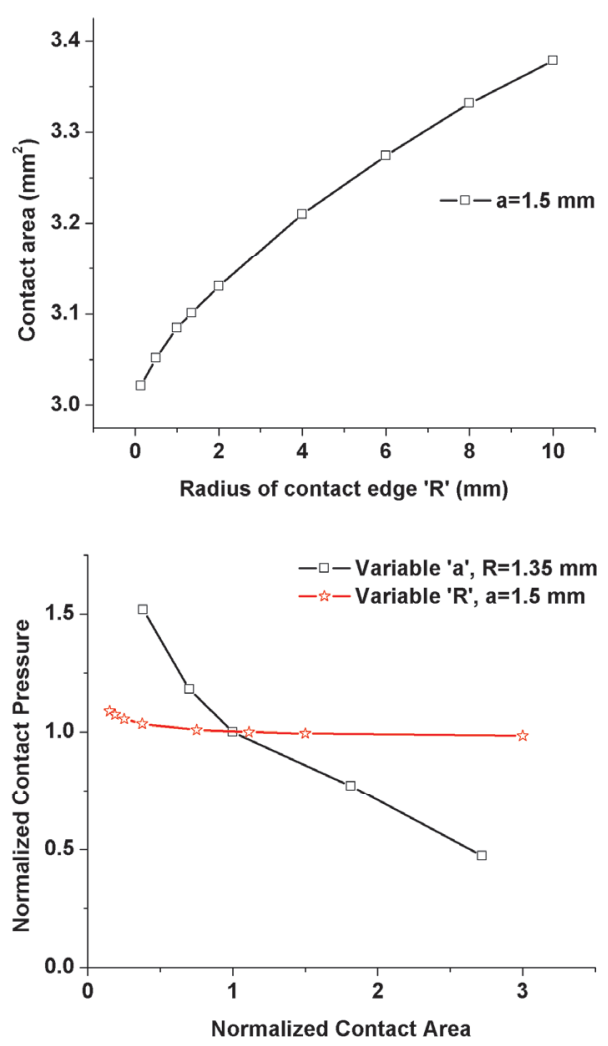

(d)

Figure 5: (a) Variation of maximum contact pressure with 'R', (b) Contact zone size variation with 'R', (c) Variation of maximum contact pressure with $\mathrm{a} / \mathrm{R}$ ratio, and (d) Normalized contact pressure variation with the normalized contact area.

(a)

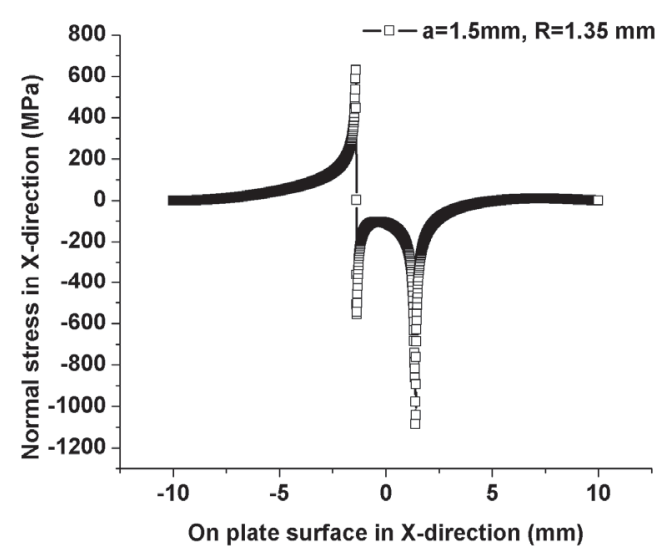

Figure 6: (a) Normal stress distribution on plate surface in X- direction, and (b) Variation of maximum normal stress (in X-direction) with $\mathrm{a} / \mathrm{R}$ ratio

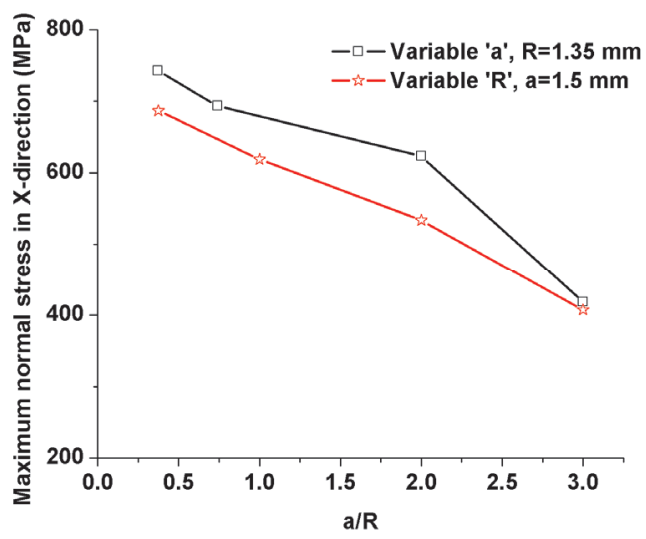

(b) 
Further, for the same normal load of $750 \mathrm{~N}$, a fully reversed tangential displacement of $15 \mu \mathrm{m}$ (triangular waveform with 5 $\mathrm{Hz}$ frequency) was applied to the flat-with-rounded edge pad in the X-direction and one fretting cycle with elasto-plastic material behavior was simulated. The normal stress distribution was extracted from plate surface after quarter stroke in the forward direction.

Fig. 6(a) shows the typical stress distribution for contact geometry with a=1.5 $\mathrm{mm}$ and $\mathrm{R}=1.35 \mathrm{~mm}$. A tensile stress peak is observed on the trailing edge which is generally considered critical from the viewpoint of crack initiation [22]. Fig. 6(b) shows the variation of peak tensile stress with a/ $R$ ratio for both the cases, viz., variable ' $a$ ', constant ' $R$ ' and variable ' $R$ ', constant ' $a$ '. It can be observed that peak tensile stress decreases with an increase in a/R ratio. At a constant value of ' $R$ ', increase in $\mathrm{a} / \mathrm{R}$ ratio means, increase in ' $\mathrm{a}$ ' i.e. half-length of the flat region, which means that as the contact geometry becomes flatter/more conformal at the center, the tendency for crack initiation decreases. Similarly, at constant 'a' value, increase in a/ $R$ ratio means, decrease in ' $R$ ' i.e. radius of contact edge, which implies that as the corners become sharper, the resistance for crack initiation increases. This is surprising because in general, filleting at the corners is considered as a measure to relieve the stresses at the contact edges but that is not always true and it may even lead to an increase in stresses. Further, it agrees with the observations reported in the literature [13, 14] where researchers have found that fretting fatigue life decreases with the increase in the corner radius and it was attributed to increased stresses due to filleting at the corners.

\section{Effect of Material Yielding}

To check whether yielding of the material has any implications on the analysis presented in the previous section, finite element analyses were also carried out using elasto-plastic material behaviour. The elasto-plastic behaviour of material was modelled using Ramberg Osgood law.

From Fig. 7(a), it can be observed that elasto-plastic analysis results deviate from elastic analysis results for lower values of $a / R$ ratio for variable ' $a$ ' and constant value of ' $R$ '. This is because, with the decrease in 'a', contact geometry starts approaching cylinder-on-plate configuration and thus leading to increased non-conformality of the contact. This results in higher stresses which lead to sufficient yielding of material and hence, elasto-plastic analysis results deviate from the elastic analysis. Further, fitting a logarithmic trend line to the contact pressure variation obtained from elastic analysis shows that as ' $a$ ' tends to zero, contact pressure approaches to that of a cylinder-on-plate case with radius ' $\mathrm{R}$ ' as $1.35 \mathrm{~mm}$.

From Fig. 7(b), it can be seen that when ' $R$ ' is varied keeping 'a' as constant, the elasto-plastic analysis results deviate from elastic analysis results at higher values of a/R ratio. This is because, with reduction in ' $R$ ', the contact geometry tends towards the flat-punch-on-half plane case, leading to highly localized stresses at the corners. Hence, the elasto-plastic analysis results deviate significantly from the elastic analysis results. Interestingly, the deviation between elastic and elastoplastic analysis results becomes significant only about $\mathrm{a} / \mathrm{R}$ value of approximately equal to one.

Further, the elastic analysis predicts higher contact pressure and stresses as compared to elasto-plastic analysis and is likely to give the conservative estimate for the fretting wear volume and fretting fatigue life which are dependent on contact pressure and tensile stress in fretting direction respectively.
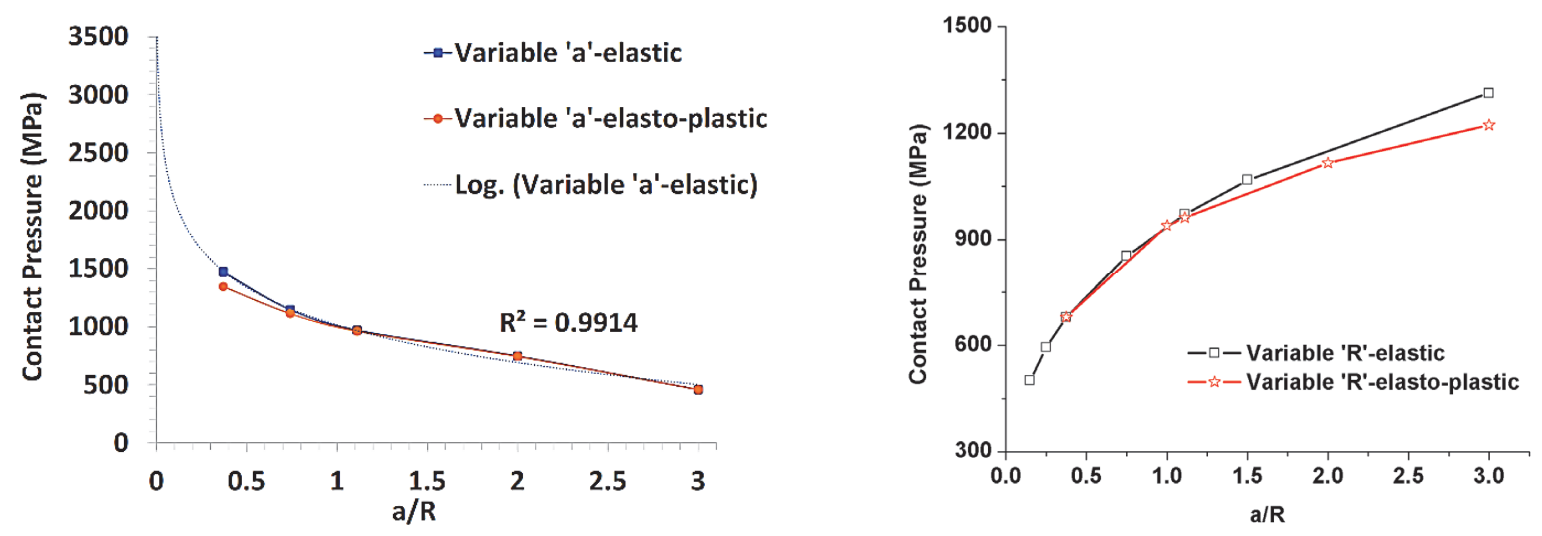

(a)

(b)

Figure 7: Effect of material yielding on the variation of maximum contact pressure with a/R ratio for (a) variable ' $a$ ' and constant 'R'=1.35 mm, and (b) variable 'R' and constant ' $a '=1.5 \mathrm{~mm}$. 


\section{CONCLUSIONS}

$\mathrm{T}$ wo-dimensional finite element analysis was carried out for a flat-with-rounded-edge contact to study the influence of contact geometry on contact tractions. The finite element analysis results were compared with the analytical results and reasonable agreement was found between them. Further, the influence of geometric parameters viz. 'a' and ' $R$ ' was studied. A comparison was also made between finite element analyses carried out using elastic and elastoplastic material behavior. Following conclusions can be drawn from the present study:

- The finite element analysis results give better correlation with analytical results for lower value of $\mathrm{a} / \mathrm{R}$ ratio.

- The effect of ' $a$ ' is more prominent on contact pressure than varying ' $R$ ' because of the greater influence of ' $a$ ' on the contact zone size.

- The effect of contact geometry on the contact stresses cannot be quantified using a unified parameter like $\mathrm{a} / \mathrm{R}$ ratio or contact area under the present loading conditions.

- Lesser filleting at the corners is found to be more beneficial as compared to generous fillets because it leads to a decrease in the peak tensile stresses. This is likely to delay the crack initiation and hence, longer fretting fatigue life. Further research is required to determine the extent of filleting which can be applied for a particular contact geometry.

- Both elastic and elasto-plastic analysis give identical results and the deviation becomes significant only when the contact geometry starts approaching either non-conformal contact or flat punch with sharp corners.

- The elastic analysis overpredicts the contact pressure and peak tensile stresses which is likely to result in a conservative estimate for fretting wear volume and fretting fatigue life.

\section{REFERENCES}

[1] Papanikos, P., Meguid, S.A., Stjepanovic, Z. (1998). Three-dimensional nonlinear finite element analysis of dovetail joints in aero-engine discs, Finite Elem. Anal. Des., 29(3-4), pp. 173-186, DOI: 10.1016/S0168-874X(98)00008-0.

[2] Söderberg, A., Andersson, S. (2009). Simulation of wear and contact pressure distribution at the pad-to-rotor interface in a disc brake using general purpose finite element analysis software, 267, pp. 2243-2251,

DOI: 10.1016/j.wear.2009.09.004.

[3] Bitter, T., Khan, I., Marriott, T., Lovelady, E., Verdonschot, N., Janssen, D. (2018). Finite element wear prediction using adaptive meshing at the modular taper interface of hip implants, J. Mech. Behav. Biomed. Mater., 77(May 2017), pp. 616-623, DOI: 10.1016/j.jmbbm.2017.10.032.

[4] Cruzado, A., Urchegui, M.A., Gómez, X. (2012). Finite element modeling and experimental validation of fretting wear scars in thin steel wires, Wear, 289, pp. 26-38, DOI: 10.1016/j.wear.2012.04.018.

[5] Kim, H.K., Lee, Y.H., Lee, K.H. (2008). On the geometry of the fuel rod supports concerning a fretting wear failure, Nucl. Eng. Des., 238(12), pp. 3321-3330, DOI: 10.1016/j.nucengdes.2008.08.010.

[6] Shinde, D.U. (2008). Wear simulation of electric contacts subjected to vibrations, Auburn University.

[7] Hills, D.A. (1994). Mechanics of fretting fatigue, 175, pp. 107-113.

[8] Fouvry, S., Paulin, C., Deyber, S. (2009). Impact of contact size and complex gross-partial slip conditions on Ti-6Al4V/Ti-6Al-4V fretting wear, Tribol. Int., 42(3), pp. 461-474, DOI: 10.1016/j.triboint.2008.08.005.

[9] Ciavarella, M., Hills, D.A., Monno, G. (1998). The influence of rounded edges on indentation by a flat punch, Inst. Mech. Engrs., 212, pp. 319-328.

[10] Ciavarella, M., Demelio, G. (2001). A review of analytical aspects of fretting fatigue, with extension to damage parameters, and application to dovetail joints, Intnl. J. Solids and Structures, 38, pp. 1791-1811.

[11] Ciavarella, M., Macina, G. (2003). New results for the fretting-induced stress concentration on Hertzian and at rounded contacts, 45, pp. 449-467, DOI: 10.1016/S0020-7403(03)00061-4.

[12] Warmuth, A.R., Pearson, S.R., Shipway, P.H., Sun, W. (2013). The effect of contact geometry on fretting wear rates and mechanisms for a high strength steel, Wear, 301(1-2), pp. 491-500, DOI: 10.1016/j.wear.2013.01.018.

[13] Juoksukangas, J., Lehtovaara, A., Mäntylä, A. (2013). The effect of contact edge geometry on fretting fatigue behavior in complete contacts, Wear, 308(1-2), pp. 206-212, DOI: 10.1016/j.wear.2013.06.013.

[14] Mall, S., Naboulsi, S., Namjoshi, S.A. (2008). Contact geometry effects on fretting fatigue crack initiation behaviour of Ti-6Al-4V, Tribol. - Mater. Surfaces Interfaces, 2(1), pp. 25-32, DOI: 10.1179/175158308X320755. 
[15] Ding, J., Leen, S.B., McColl, I.R. (2004). The effect of slip regime on fretting wear-induced stress evolution, Int. J. Fatigue, 26(5), pp. 521-531, DOI: 10.1016/j.ijfatigue.2003.09.001.

[16] Ambrico, J.M., Begley, M.R. (2000). Plasticity in fretting contact, J. Mech. Phys. Solids, 48(11), pp. 2391-417.

[17] Abaqus 6.12-1 documentation.

[18] James, L.A., Steel, A.A. (2017). Ramberg-Osgood Strain- Hardening Characterization, J. Pressure Vessel Technology 117 , pp. 341-345.

[19] Anandavel, K., Prakash, R. V. (2011). Effect of three-dimensional loading on macroscopic fretting aspects of an aeroengine blade - disc dovetail interface. Tribiology Int., 44(11), pp. 1544-1555, DOI: 10.1016/j.triboint.2010.10.014.

[20] Hertz, H. (1882). On the contact of elastic solids, J. Pure and Applied Mathematics, 92, pp. 156-171.

[21] K. L. Johnson. (1985). Contact Mechanics, Cambridge, Cambridge University Press.

[22] McVeigh, P. a., Farris, T.N. (1997). Finite Element Analysis of Fretting Stresses, J. Tribol., 119(4), pp. 797, DOI: $10.1115 / 1.2833887$. 